\title{
Bronchial compliance and wall structure during development of the immature human and pig lung
}

\author{
P.K. McFawn, H.W. Mitchell
}

Bronchial compliance and wall structure during development of the immature human and pig lung. P.K. McFawn, H.W. Mitchell. (CERS Journals Ltd 1997.

ABSTRACT: Maturational changes in the specific compliance could potentially contribute to the development or clinical presentation of respiratory diseases in infants and children. Changes in the specific compliance during development and its structural basis have been well characterized, but changes in bronchial compliance and the mechanisms involved have received little attention.

Semistatic pressure-volume curves were generated for isolated bronchial segments from late-term foetal, immature and adult pigs. A small number of bronchi from human infants were also studied. The amount of cartilage in the bronchial wall of pigs of different ages was measured histologically, and morphometric changes in the wall of inflated bronchi were investigated.

The specific compliance of bronchi approximately halved from 1 to 4 weeks of age. No change in specific compliance was observed either between 4 week old and adult pigs, or between late-term foetal and 1 week old pigs. Changes in the total wall and cartilage areas did not correlate with changes in specific compliance. Inflation to $20 \mathrm{cmH}_{2} \mathrm{O}$ transmural pressure reduced the total wall area of bronchi from 1 week old pigs.

Significant changes in bronchial distensibility occur during the early postnatal period. These changes in specific compliance are not caused by an increase in the amount of cartilage. The increase in luminal volume during inflation of bronchial segments occurs, partially, by compression of the airway wall against the cartilage layer.

Eur Respir J., 1997; 10: 27-34.

Specific compliance is a measure of distensibility, which reflects how easily an airway can deform. Numerous forces acting on the airway wall, including transmural pressure and smooth muscle contraction, may distend or narrow conducting airways [1-4]. Compliant airways are more susceptible both to distension and compression by these forces.

Increased airway compliance is associated with several diseases, including tracheobronchomegaly and some forms of chronic obstructive pulmonary disease (COPD) [3]. Airway rigidity (which is inversely proportional to specific compliance) influences the extent of smooth muscle contraction and airway narrowing by contributing both to the pre- and afterload acting on the muscle [5, 6]. IsHida et al. [7], Moreno et al. [8] and BRAMLEY et al. [9] have speculated that reducing the afterload (which limits shortening $[6,10]$ ) on airway smooth muscle could produce the hyperresponsiveness seen in asthma. The wave speed theory of expiratory flow limitation implies that maximum expiratory flow is dependent on airway compliance [11]. Increasing large airway compliance increases airway resistance and decreases maximum expiratory flow $[3,12]$, which could contribute to the airway obstruction associated with COPD [3].

Maturational changes in compliance could influence respiratory diseases of infancy and childhood, such as childhood asthma, bronchopulmonary dysplasia and acquired tracheomegaly $[2,13]$. Compliant immature
Dept of Physiology, The University of Western Australia, Nedlands, Australia.

\section{Correspondence: P.K. McFawn}

Dept of Physiology

The University of Western Australia Nedlands 6907

Australia

Keywords: Airway compliance bronchi

lung development

Received: September 111995

Accepted after revision August 201996

This research was supported by the Sudden Infant Death Syndrome Foundation of Western Australia, the Australian National SIDS Council and the Raine Foundation of Western Australia. bronchi could be more vulnerable to collapse during forced expiration, decreasing maximum expiratory flow. Peripheral airway collapse has been suggested as a factor contributing to sudden infant death syndrome (SIDS) $[14,15]$, and collapse is more likely to occur if the bronchi are highly compliant. However, the compliance of bronchi both from healthy infants and SIDS cases is unknown.

Maturational changes in tracheal pressure-volume curves have been extensively studied, and show a progressive decrease in tracheal compliance from the early foetus to the adult $[2,16-18]$. However, the bronchi are more physiologically relevant to many diseases than the trachea; furthermore, bronchi are structurally different to the trachea with the smooth muscle separated from the cartilage by a region of connective tissue. No systematic study of bronchial compliance has been made concerning the perinatal and neonatal periods.

Previously, we have shown that, during inflation, the specific compliance of bronchi of adult and 4 week old pigs is the same [19]. The aim of this study was to determine whether there are maturational changes in bronchial compliance over the prenatal period, up to 4 weeks of postnatal life. Specific compliance was measured by constructing normalised volume (strain)-pressure curves for bronchial segments of late term foetal, 1 week and 4 week old pigs. The effect of airway location (i.e. generation) on the strain-pressure curves was investigated 
by comparing proximal and distal bronchial segments from pigs of the same age. A small number of human infant bronchi (from SIDS cases) were also studied. Morphometric techniques were used to determine whether changes in the amount of bronchial cartilage or wall thickness were associated with increased rigidity. An attempt was made to identify the histological regions of the bronchial wall important in determining specific compliance by examining morphological changes of inflated versus uninflated airways.

\section{Methods}

\section{Preparation of bronchial segments}

Porcine lungs were obtained from freshly killed adult, 1 and 4 week old pigs (Large White/Landrace cross). Late-term foetal pig ( $>100$ days of the 115 day gestation in the pig) lungs were supplied by a local abattoir. Segments of stem bronchus, approximately $25 \mathrm{~mm}$ long, were dissected from the lower lobes as described previously [20]. Airway generation was measured by counting the number of side branches off the stem bronchus, taking the trachea as generation zero. The internal diameter of the bronchial segments was measured at the distal end using steel rods gently inserted into the first $2 \mathrm{~mm}$ of the lumen. A small number of human lungs from SIDS autopsies were obtained from the Western Australian State Coroner during 1990-1992. Bronchial segments from SIDS cases were of lobar-segmental bronchi, but exact generation was difficult to assess and the length and internal diameter varied depending on the tissue available.

Bronchial segments were cannulated at both ends with polyethylene tubing and mounted horizontally in a modified organ bath [21] filled with warm $\left(37^{\circ} \mathrm{C}\right) \mathrm{Krebs}$ solution, aerated with $5 \% \mathrm{CO}_{2}$ in $\mathrm{O}_{2}$. The lumen of the segment was bathed with Krebs solution and regularly flushed with warm aerated Krebs solution. The bronchial lumen was connected to a pressure transducer (Motorrola, Phoenix, AZ, USA) to measure transmural pressure and to microsyringe for changing luminal volume. The bronchi were tested for leaks by connecting the lumen to a $10 \mathrm{cmH}_{2} \mathrm{O}$ pressure head for $10-15 \mathrm{~min}$. The tap leading to the pressure head was then closed and the luminal pressure monitored for $10 \mathrm{~min}$. Isolated foetal and adult pig airways do not generate spontaneous active tone [22]. Hence, all experiments were performed with the bronchial smooth muscle relaxed.

\section{Compliance curves}

Semistatic compliance curves were generated by changing the luminal volume in $0.02 \mathrm{~mL}$ increments and recording the luminal pressure. After each volume increment, pressure was allowed to equilibrate until $>80 \%$ of the stress relaxation had occurred (2-3 min). Bronchial segments from adult, 4 and 1 week old pigs were inflated to a transmural pressure of approximately $+30 \mathrm{cmH}_{2} \mathrm{O}$; bronchi from SIDS cases and foetal pigs were inflated to only $+20 \mathrm{cmH}_{2} \mathrm{O}$ approximately. Following inflation, bronchi were deflated until a transmural pressure of ap- proximately $-25 \mathrm{cmH}_{2} \mathrm{O}$ was reached. Two complete pressure-volume loops were performed on each bronchus to standardize volume history before recording a third pressure-volume cycle, which was used for analysis.

Previous studies of isolated airway compliance have normalized for differences in airway size by using either the initial volume $[2,16,18,19]$, or the volume at some arbitrarily chosen maximum pressure [5, 23]. For the most part, we chose to normalize compliance for the lumen volume at $0 \mathrm{cmH}_{2} \mathrm{O}\left(V_{0}\right)$ as this method has been used to study developmental changes in the specific compliance of the trachea $[2,16,18]$. Lumen volume (at 0 $\mathrm{cmH}_{2} \mathrm{O}$ ) was measured as described previously [19]. The segments were removed from the organ bath and emptied by blowing air through the lumen using a syringe. The bronchial segments were then filled with Krebs solution and the volume required to fill the segment recorded. The measurement of lumen volume was repeated three times on each segment and the mean taken. The coefficient of variation ranged $5-11 \%$, smaller than for alternative methods, such as weighing the full and empty bronchus (29-65\%). Lumen volume calculated from cross-sectional area and length was similar to the measured volume.

To determine whether the method of normalizing volume change altered the results, the compliance of foetal, 1 and 4 week old pig bronchi was also normalized for the lumen volume at an intraluminal pressure of 20 $\mathrm{cmH}_{2} \mathrm{O}(V 20)$. The volume at $20 \mathrm{cmH}_{2} \mathrm{O}$ was calculated for each bronchus by fitting the equation $V=\mathrm{A}-\mathrm{Be}^{-\mathrm{k} P}$ to the inflation data (according to the method of Moreno et al. [4]) where $V$ is volume, $P$ is pressure, A, B and $\mathrm{k}$ are constants. A good fit was obtained to the data and $\mathrm{r}^{2}$ ranged 0.92-0.99.

Numerical values for compliance were calculated by plotting strain or $\% V 20$ against pressure for each bronchus. The coefficient of determination was calculated and data points removed from the ends of the curve until $\mathrm{r}^{2}$ was greater than 0.9 , this was taken as the linear region of the pressure-volume curve. The slope of the linear region was then calculated by least squares regression. Compliance was calculated separately for each bronchi's inflation and deflation pressure-volume curve.

\section{Morphology}

Bronchi used for morphological examination were fixed either at 0 or $+20 \mathrm{cmH}_{2} \mathrm{O}$ transmural pressure in phosphate-buffered formalin saline. After fixation, bronchi were washed in phosphate-buffered saline and embedded in CRYO-M-BEDTM (Bright, Huntingdon, UK) for sectioning with a cryostat. Cryoprocessing was used to minimize tissue shrinkage. Sections were cut $15 \mu \mathrm{m}$ thick, stained with alcian blue (Gurr; BDH, Poole, UK) and safranine $\mathrm{O}(\mathrm{BDH})$, air dried, dipped in histoclear (Ajax, Auburn, Australia) and mounted, without washing or nuclear counterstaining. Sections were digitized and analysed using a CCD camera (ICD-8HOR RGB; Ikegami Tsushinki, Japan) and image processing work station (Video Pro; Leading Edge, Adelaide, Australia).

Two image processing protocols were used, one to compare the amount of cartilage in bronchi from different generations or ages and the other to quantify the effect of inflation on airway size and shape. Separate 
specimens were used to examine the effects of age, generation and inflation on the airway wall. Histological sections used to study the effect of age on airway morphology came from the distal end of bronchial segments used to measure compliance. Duplicate sections were measured from each specimen used. In the first protocol the outer $(\mathrm{Ao})$, internal $(\mathrm{Ai})$ and cartilage areas (WAcart) were measured on sections fixed at $0 \mathrm{cmH}_{2} \mathrm{O}$. Total wall area (WAtot) and the percentage of cartilage in the wall $(\%$ Cart=WAcart/WAtot $\times 100)$ were then calculated. In the second protocol, the internal and outer perimeters and areas $(\mathrm{Pi}, \mathrm{Po}, \mathrm{Ai}, \mathrm{Ao})$ were measured on segments from adult and 1 week old pigs fixed at 0 and $+20 \mathrm{cmH}_{2} \mathrm{O}$. The ratio between the internal area calculated $(\mathrm{CAi})$ from $\mathrm{Pi}$ and the measured value of $\mathrm{Ai}$ was used as an index of bronchial circularity $(\mathrm{CAi} / \mathrm{Ai})$. In a few uninflated adult bronchi, the ratio between the longest and shortest internal "diameters" was also calculated, and this ranged $1.05-1.76($ average $=1.38 ; n=4)$. The nomenclature and abbreviations of BAI et al. [24], are used, in this study, for bronchial morphology.

\section{Solutions}

The Krebs solution used had the following composition (in $\mathrm{mM}$ ): $121 \mathrm{NaCl}, 5.4 \mathrm{KCl}, 1.2 \mathrm{MgSO}_{4}, 1.2$ $\mathrm{NaH}_{2} \mathrm{PO}_{4}, 25 \mathrm{NaHCO}_{3}, 11.5$ glucose, $2.5 \mathrm{CaCl}_{2}$. The phosphate-buffered saline had the following composition (in $\mathrm{mM}$ ): $150 \mathrm{NaCl}, 5 \mathrm{NaH}_{2} \mathrm{PO}_{4}, 15 \mathrm{Na}_{2} \mathrm{HPO}_{4}$, and was buffered to $\mathrm{pH}$ 7.2. The fixative was $10 \%$ formalin in phosphate-buffered saline. The alcian blue and safranine stain contained $0.36 \%$ alcian blue, $0.18 \%$ safranin $\mathrm{O}$ and $0.48 \%$ ferric ammonium sulphate, dissolved in an acetate $(0.2 \mathrm{M})$ and $\mathrm{HCl}(0.24 \mathrm{M})$ buffer at $\mathrm{pH}$ 1.5. The drugs used were acetylcholine chloride (Sigma, St. Louis, USA), isoproterenol hydrochloride (isoprenaline, Sigma) and salbutamol sulphate (Glaxo Wellcome, Stevenage, UK).

\section{Statistics}

Specific compliance was obtained from the linear region of the normalized volume-pressure curves using simple two-stage regression analysis [25]. Significance bet- ween multiple groups was tested by one-way analysis of variance (ANOVA) with Student-Newmann-Keuls post hoc test. When only two groups were compared, Student's t-test was used to determine significance. All results are reported as mean \pm standard error of the mean, with a p-value of less than 0.05 considered to be significant. The morphological data showed a high variance so the minimum difference in the means that could be reliably detected $(\beta<0.1)$ was calculated from the sample size and standard deviation. At least four pigs were used for all experiments, with " $n$ " indicating the total number of segments.

\section{Results}

The size (luminal volume and distal end diameter) and location along the stem bronchus (generation) of the segments studied together with their specific compliances during inflation and deflation are shown in table 1. No difference was found between the deflation specific compliance of adult and 4 week old bronchial segments. In a previous study [19], we showed that during inflation the specific compliance of adult and 4 week old pigs were the same. In contrast, bronchi from 1 week old pigs were approximately twice as distensible as those from 4 week old pigs (fig. 1a and table 1). Pressure-strain curves for late-term foetal and 1 week old pigs were similar (fig. $1 \mathrm{~b}$ and table 1). Similar differences in specific compliance were found among foetal, 1 and 4 week old bronchi, whether the volume change was normalized as strain $\left(\Delta V / V_{0}\right)$ or $\% V 20$. Inflation and deflation specific compliances were not different for any age group studied (table 1 ). The ratio of the resting to maximum volume $\left(V_{0} / V_{20}\right)$ was significantly less in foetal and 1 week old bronchi compared to 4 week old pigs (table 1 ). The $V_{0} / V_{20}$ ratio was the same for bronchi from foetal and 1 week old pigs, and for bronchi from 4 week old pigs compared to adults.

The effect of altering position along the stem bronchus (generation) on specific compliance was examined using distal and proximal segments of stem bronchus from 4 week old pigs, and by histologically investigating changes in bronchial structure. Distal bronchial segments were taken from generations 9-15 (proximal to

Table 1. - Age comparison: location, size and specific compliance of bronchial segments from pigs of different ages

\begin{tabular}{|c|c|c|c|c|c|c|c|c|c|}
\hline \multirow{2}{*}{$\begin{array}{l}\text { Age } \\
\text { weeks }\end{array}$} & \multirow{2}{*}{$\begin{array}{l}\text { Length } \\
\mathrm{mm}\end{array}$} & \multirow{2}{*}{$\begin{array}{c}\text { Internal } \\
\text { diameter } \\
\mathrm{mm}\end{array}$} & \multirow{2}{*}{$\begin{array}{l}\text { Gene- } \\
\text { ration }\end{array}$} & \multirow{2}{*}{$\begin{array}{l}\text { Lumen } \\
\text { volume } \\
\text { mL }\end{array}$} & \multicolumn{2}{|c|}{ Inflation compliance } & \multicolumn{2}{|c|}{ Deflation compliance } & \multirow[t]{2}{*}{$\% V_{0} / V_{20}$} \\
\hline & & & & & as strain & as $\% V 20$ & as strain & as $\% V 20$ & \\
\hline Foetal $(n=10)$ & 25 & $1.8 \pm 0.04$ & $1-10$ & $0.051 \pm 0.004$ & $0.10 \pm 0.009$ & $3.99 \pm 0.30$ & $0.14 \pm 0.013$ & $5.26 \pm 0.40$ & $39.8 \pm 1.3$ \\
\hline One $(n=10)$ & 25 & $1.9 \pm 0.04$ & $2-12$ & $0.063 \pm 0.004$ & $0.10 \pm 0.015$ & $4.17 \pm 0.42$ & $0.13 \pm 0.017$ & $5.18 \pm 0.57$ & $39.4 \pm 1.3$ \\
\hline Four $(n=8)$ & 25 & $2.0 \pm 0.03$ & $8-15$ & $0.078 \pm 0.005$ & $0.055 \pm 0.005^{*}$ & $2.49 \pm 0.16^{*}$ & $0.055 \pm 0.004 * * *$ & $2.85 \pm 0.16^{* * *}$ & $* 50.6 \pm 1.3 * * *$ \\
\hline Four & 35 & $2.6 \pm 0.07$ & - & $0.18 \pm 0.01$ & $0.064^{\#}$ & - & 0.064 & - & $55.2 \pm 2.9$ \\
\hline Adult $(\mathrm{n}=5)$ & 35 & $2.4 \pm 0.08$ & - & $0.2 \pm 0.01$ & $0.061^{\#}$ & - & $0.055 \pm 0.023$ & - & $50.5 \pm 3.0$ \\
\hline
\end{tabular}

Bronchial segments of the same distal internal diameter were compared to examine the effect of age on specific compliance. Internal diameter was kept constant with age by selecting segments from different locations (generation) along the stem bronchus. Segment generation is from the proximal to the distal end of the segment, counting the trachea as generation zero. Luminal volume $\left(V_{0}\right)$ is the volume of the bronchial segment lumen with no transmural pressure difference. The change in lumen volume during inflation and deflation was normalized by calculating the strain (change in volume $(\Delta V) / V 0)$, or as a percentage of the volume at $+20 \mathrm{cmH}_{2} \mathrm{O}$ intraluminal pressure $(\% \mathrm{~V} 20)$. Inflation and deflation compliances refer to the slope of the linear region of the specific compliance of the normalized volume-pressure curves expressed as either the change in strain/ $\mathrm{cmH}_{2} \mathrm{O}$ or $\% V_{20} / \mathrm{cmH}_{2} \mathrm{O}$. The ratio between the lumen volume at 0 and $+20 \mathrm{cmH}_{2} \mathrm{O}$ is shown as $\% V 0 / V 20$. *: $\mathrm{p}<0.05$; ***: $\mathrm{p}<0.001$, compared to foetal and 1 week. (\#: from [20]). 
a)

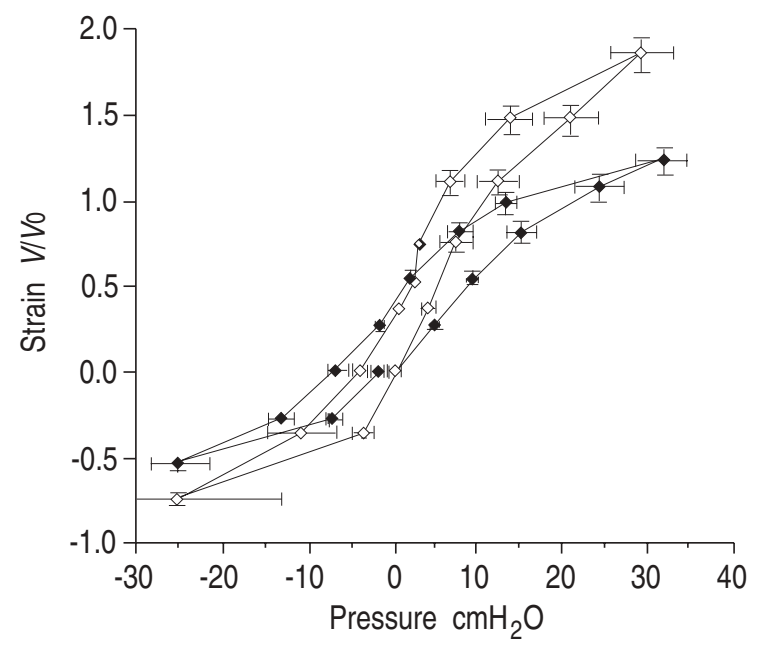

b)

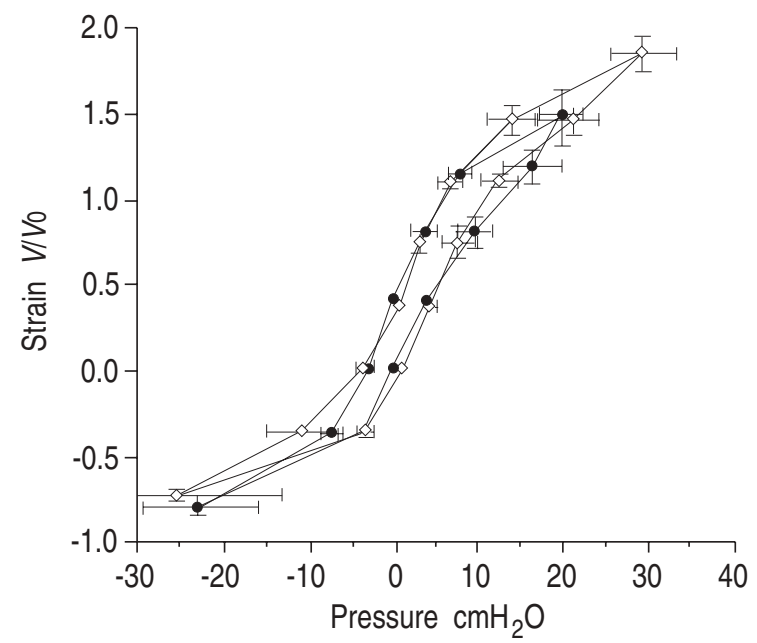

Fig. 1. - Pressure-strain (specific compliance) curves for pig bronchial segments of the same internal diameter (at the distal end) from different ages. Strain $\left(\Delta V / V_{0}\right)$ was calculated from the change in volume divided by the volume at $0 \mathrm{cmH}_{2} \mathrm{O}$; i.e. a strain of 0 represents the initial volume and a strain of 1 represents twice the initial volume. The slope of the linear region was used to measure compliance during inflation and deflation (table 1). a) Bronchial segments from $1(\mathrm{n}=10)$ and $4(n=8)$ week old pigs. Segments from 1 week old pigs were significantly more complaint than those from 4 week olds $(\mathrm{p}<0.05)$. b) Compliance curve for segments of late-term foetal ( $>100$ days of 115 day gestation) pig bronchi $(n=10)$ with that of 1 week old pig bronchi $(\mathrm{n}=10$; data from panel a). $\longrightarrow-1$ : 1 week old pigs; $\longrightarrow \longrightarrow$ : 4 week old pigs; $\longrightarrow$ : foetal pigs. Error bars indicate one standard error of the mean. distal end of the segment) corresponding to the location of the 4 week old bronchial segments used previously. Proximal bronchial segments were taken from generations 2-8, which was similar to the location of the 1 week old segments used (tables 1 and 2). Pressure strain curves for these airways were similar and the values of linear compliance measured were not significantly different (fig. 2 and table 2). Furthermore, the percentage of cartilage (\% Cart) in the stem bronchus of 4 week old pigs was constant from generation 2 to 23 (fig. 3). No qualitative differences were observed in the structure of the stem bronchus in histological sections from the 2nd to 23rd generation.

Airway morphology was investigated in an attempt to identify structural changes that might produce the observed maturation of specific compliance. Bronchi from 1 week old pigs had a significantly lower total wall area (WAtot) and cartilage area (WAcart) than those from foetal and 4 week old pigs $(\mathrm{p}<0.01)$ (fig. 4). However, the percentage of cartilage (\% Cart) in the wall of foetal $(34 \pm 1.5 \%), 1(42 \pm 2.8 \%)$ and $4(40 \pm 2.6 \%)$ week old pigs was the same.

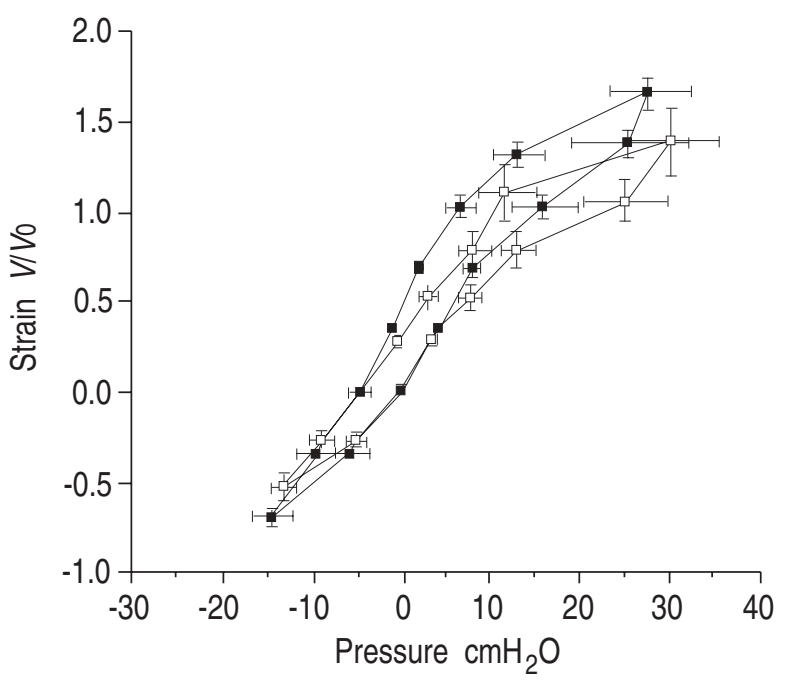

Fig. 2. - Specific compliance curves for proximal $(n=6)$ and distal $(n=6)$ segments of main stem bronchus from 4 week old pigs. Distal bronchial segments were from generations 9-15 (proximal-distal end of the segment), whilst proximal segments came from generations $2-8$. The bronchial generations used for proximal and distal segments, respectively, were similar to the generations used for the 1 week and 4 week old segments in figure 1 . There was no significant difference in compliance between proximal and distal segments (table $2)$. $\square$ : distal segment; $\square-$ : proximal segments. Error bars indicate one standard error of the mean.

Table 2. - Location, size and specific compliance of distal and proximal segments of stem bronchus from 4 week old pigs

\begin{tabular}{llccccc}
\hline Generation & $\mathrm{n}$ & Length & $\begin{array}{c}\text { Distal internal } \\
\text { diameter } \\
\mathrm{mm}\end{array}$ & $\begin{array}{c}\text { Lumen } \\
\text { volume } \\
\mathrm{mL}\end{array}$ & $\begin{array}{c}\text { Inflation } \\
\text { compliance } \\
\text { as strain }\end{array}$ & $\begin{array}{c}\text { Deflation } \\
\text { compliance } \\
\text { as strain }\end{array}$ \\
\hline $9-15$ & 6 & 25 & $2.0 \pm 0.03$ & $0.057 \pm 0.007$ & $0.076 \pm 0.007$ & $0.089 \pm 0.01$ \\
$2-8$ & 6 & 25 & $3.2 \pm 0.11$ & $0.14 \pm 0.02$ & $0.064 \pm 0.009$ & $0.066 \pm 0.01$
\end{tabular}

The specific compliance of airways from bronchi of the same age but from different locations (generations) was compared as a control for changing airway generation between ages (see table 1). Bronchial segments were selected to have generations similar to those used previously for 1 and 4 week old pigs. Segment generation is from the proximal to the distal end of the segment, counting the trachea as generation zero. Luminal volume $\left(V_{0}\right)$ was measured when there was no transmural pressure difference. Inflation and deflation compliances refer to the specific compliance of the normalized volume-pressure curves. n: number of bronchial segments. Volume changes $(\Delta V)$ were normalized for $V_{0}$ as strain (i.e. $\Delta V / V 0)$ 


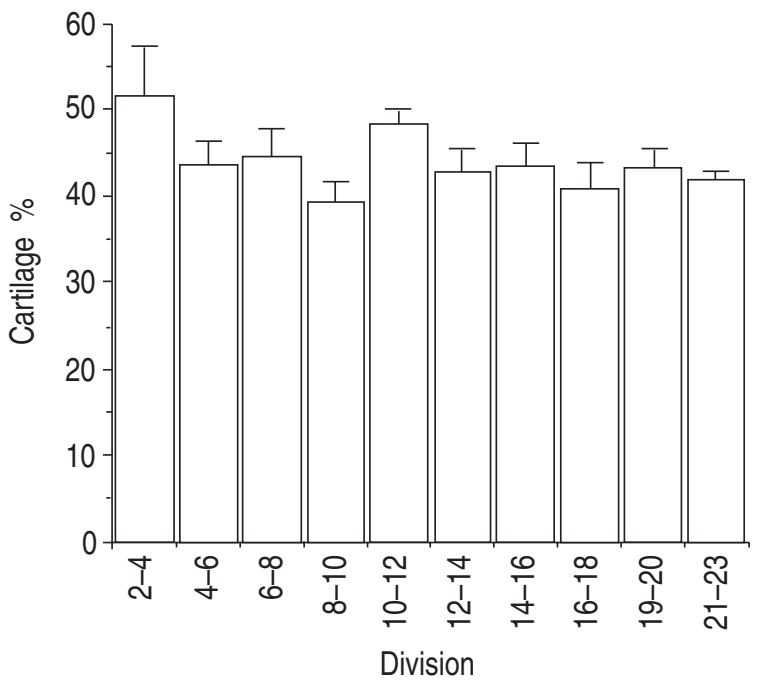

Fig. 3. - The proportion of cartilage in the wall of bronchial segments from different regions of the stem bronchus of 4 week old pigs $(n=5)$. The proportion of cartilage was constant throughout the length of the stem bronchus. Error bars indicate one standard error of the mean.

Adult and 1 week bronchial segments were examined morphometrically to determine where deformation occurs during inflation and the histological regions that could be important for determining bronchial distensibility. The internal diameter (determined from $\mathrm{Pi}$ ) of uninflated adult bronchi was $2.8 \pm 0.1 \mathrm{~mm}$, while uninflated 1 week old bronchi had a diameter of $2.4 \pm 0.1$ $\mathrm{mm}$. The outer and internal perimeters $(\mathrm{Po}, \mathrm{Pi})$ of both ages were unaffected by inflation. Inflation produced a significant increase in internal area $\left(\mathrm{Ai}_{\mathrm{i}}\right)$ of bronchi both of adult and 1 week old pigs. In both age groups, there was a trend for outer area (Ao) to increase and total wall area (WAtot) to decrease with inflation. In 1 week old bronchi, WAtot was significantly reduced by inflation (fig. 5). No significant changes in Ao could

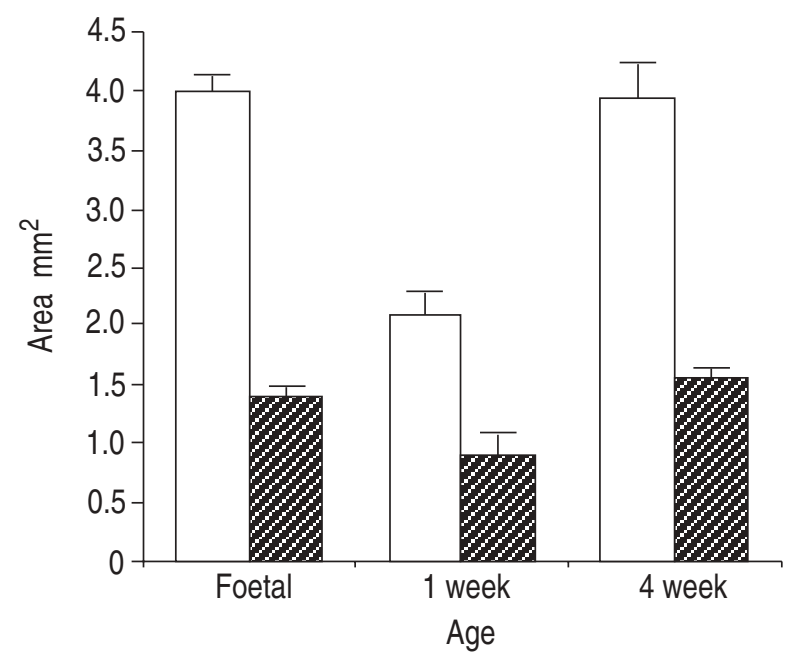

Fig. 4. - Total wall area (WAtot) and cartilage area (WAcart) of bronchial segments from late-term foetal $(n=10), 1(n=4)$ and four $(n=5)$ week old pigs fixed at atmospheric pressure (internal diameter of approximately $2 \mathrm{~mm}$ ). The proportion of cartilage in the bronchial wall was the same for all three age groups. However, segments from 1 week old pigs had a reduced WAtot and WAcart compared both to foetal and 4 week old bronchi $(\mathrm{p}<0.05)$. There was no different in WAtot or WAcart between foetal and 4 week old bronchial segments. $\square$ : total wall area; $\square$ : cartilage area. Error bars indicate one standard error of the mean. be detected after inflation of bronchi from adult or 1 week old pigs. The ratio of internal area calculated from $\mathrm{Pi}$ on the measured internal area $(\mathrm{CAi} / \mathrm{Ai})$ decreased from $1.57 \pm 0.12$ to $1.35 \pm 0.05$ (Ns) with inflation of bronchi from 1 week old pigs. Adult bronchi had a $\mathrm{CA}$ / $/ \mathrm{Ai}$ ratio of $1.59 \pm 0.08$ uninflated and $1.28 \pm 0.02$ inflated $(\mathrm{p}<0.05)$, indicating that inflated bronchi are more circular.

Figure 6 compares the histological appearance of uninflated and inflated bronchial segments from 1 week old pigs. Uninflated bronchi show deep mucosal folds and a small submucosal space between the smooth muscle and cartilage. Mucosal folds are shallow or absent in inflated bronchi and the airway wall luminal to the cartilage is thinner.

Strain-volume curves for human bronchi from five cases of SIDS were also determined. All of these airways were cartilaginous at their distal ends. The distal
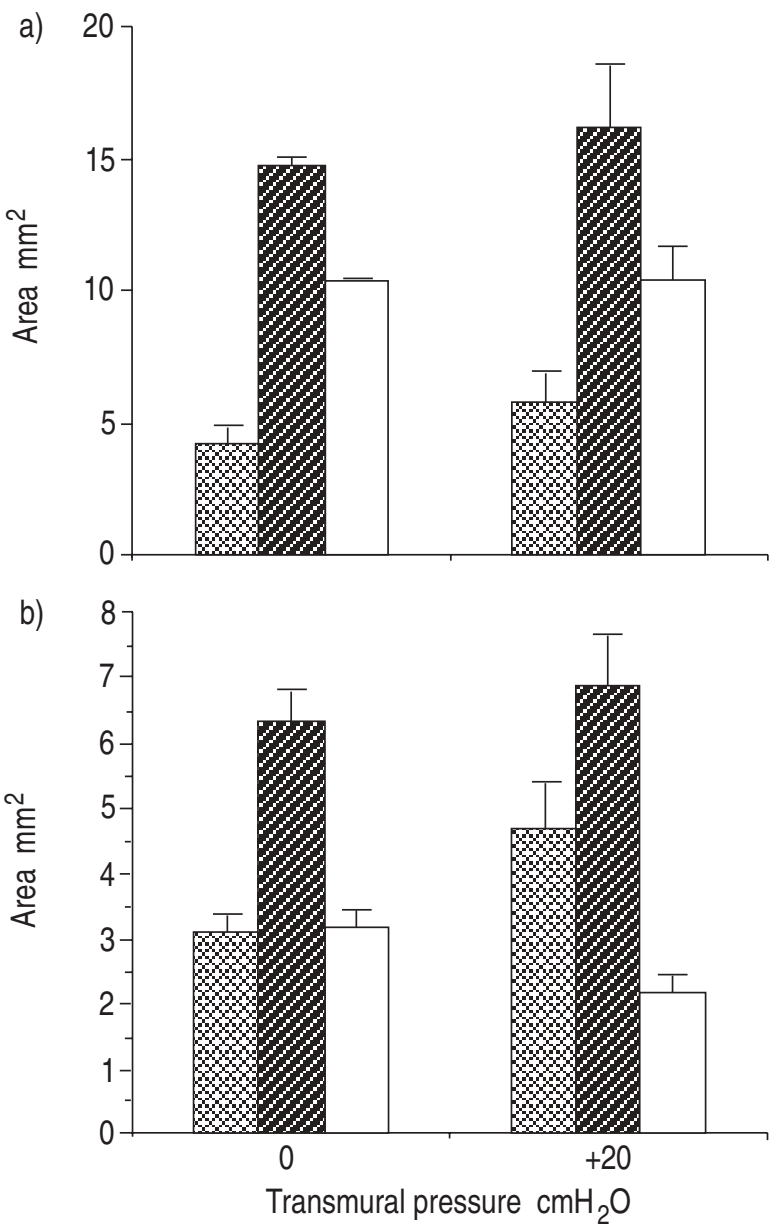

Fig. 5. - Effect of airway inflation on wall morphology of bronchi from: a) adult; and b) 1 week old pigs. a) internal area ( $\square$ ), outer area $(\square)$ and total wall area $(\square)$ of uninflated $\left(0 \mathrm{cmH}_{2} \mathrm{O} ; \mathrm{n}=6\right)$ and inflated $\left(20 \mathrm{cmH}_{2} \mathrm{O} ; \mathrm{n}=7\right)$ bronchi from adult pigs. Internal area was significantly increased by inflation $(\mathrm{p}<0.05)$. However, no statistically significant difference in outer area or total wall area was detected after inflation. Internal perimeters were $8.71 \pm 0.22$ and $9.84 \pm 0.51$ $\mathrm{mm}$ for uninflated and inflated bronchi, respectively. b) Internal area, outer area and total wall area of uninflated $\left(0 \mathrm{cmH}_{2} \mathrm{O} ; \mathrm{n}=6\right)$ and inflated $\left(20 \mathrm{cmH}_{2} \mathrm{O} ; \mathrm{n}=6\right)$ bronchi from 1 week old pigs. The outer area of bronchi from 1 week old pigs did not show a statistically significant change after inflation. Internal area was significantly increased $(p<0.05)$ and total wall area decreased $(p<0.05)$ by inflation of 1 week old bronchi. Uninflated bronchi had an internal perimeter of $7.66 \pm$ $0.24 \mathrm{~mm}$, whilst inflated bronchi had an internal perimeter of $8.76 \pm 0.58$ $\mathrm{mm}$. Error bars indicate one standard error of the mean. 

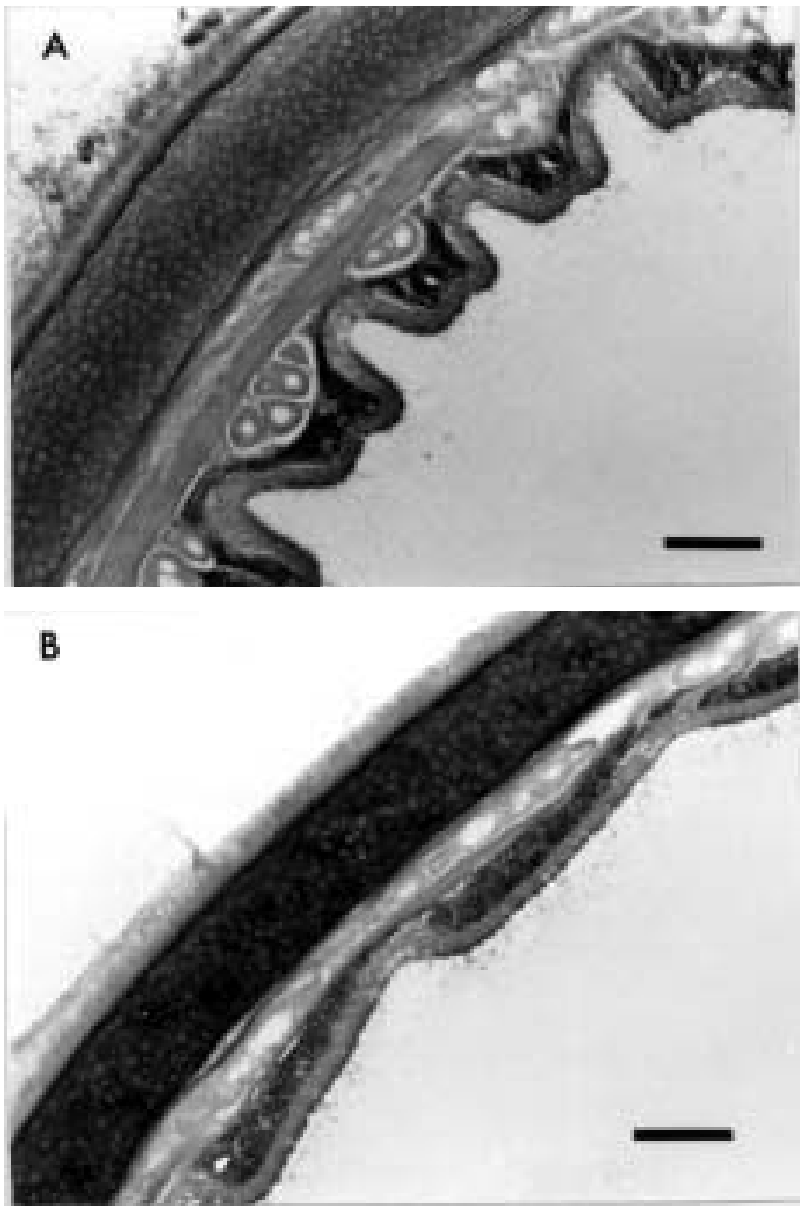

Fig. 6. - Photomicrographs of: A) uninflated and B) inflated bronchi from 1 week old pigs. Bronchi were inflated by a transmural pressure of $20 \mathrm{cmH}_{2} \mathrm{O}$, and then fixed. Uninflated bronchi show a more folded epithelium and a thicker airway wall compared to inflated bronchi. (Alcian blue and safranine stain; internal scale bar $=100 \mu \mathrm{m}$ ).

internal diameter, luminal volume $\left(V_{0}\right)$ and linear compliance of each segment is given in table 3. Specific compliance of the human bronchi was similar to that of the 1 week old pigs studied, and no difference was seen between the inflation and deflation limbs of the compliance curves. Three of the SIDS bronchi were challenged with isoprenaline $(300 \mu \mathrm{M})$ and/or salbutamol $(30 \mu \mathrm{M})$, neither of which produced any relaxation. No bronchi from SIDS cases were responsive to $300 \mu \mathrm{M}$ acetylcholine.

\section{Discussion}

We observed a large decrease in bronchial distensibility between 1 and 4 weeks of age, but there was little or no change during the last few weeks of gestation. This pattern of development, in pig bronchi, contrasts with rabbit trachea, in which there is a continuous reduction of tracheal compliance during foetal development [16]. However, in both species the mechanisms that determine specific compliance are fully established by approximately 4 weeks of extrauterine life ([16, 19], and present study).

Bronchi from immature foetal and 1 week old pigs had an initial volume which was a significantly smaller fraction of their inflated volume $(\% V 0 / V 20)$ than found in older animals. This difference in $\% V 0 / V 20$ could be due to the higher compliance of the immature animals. Alternatively, the maximum inflation of the older bronchi may be limited independently of their decreased linear compliance. Treatment of lungs with elastase greatly increases compliance over the linear region of the pressure-volume curve with no effect on maximum volume, while collagenase increases the maximum inflation without altering compliance [26]. We suggest that both the specific compliance and the maximum inflation of isolated bronchi decrease with maturation.

Human tracheal compliance decreases progressively from infancy to adulthood [18], but comparable changes in bronchi from infants or children have not been observed. The infant bronchi in this study were approximately twice as compliant as the 4-11 year old children (and single infant) studied by CROTEAU and CoOK [18]. The different specific compliances measured in the present study and in that of CROTEAU and CoOK [18] may suggest a maturational change in human bronchi similar to that characterized for trachea, although it could be due to differences in the airway generations used between the two studies. We found similar values of specific compliance both for human infant and 1 week old pig bronchi.

Because of lung growth during development, bronchi of both the same size and generation cannot be compared among different ages. In this study, bronchi of similar internal diameter and length were compared, but this meant that segments from younger animals were taken from more proximal regions of the stem bronchus (table 1). However, the proportion of cartilage in the bronchial wall was constant along the stem bronchus and no

Table 3. - Human bronchi: size and specific compliance of human bronchial segments

\begin{tabular}{lcccc}
\hline $\begin{array}{l}\text { Bronchial } \\
\text { segment }\end{array}$ & $\begin{array}{c}\text { Distal internal } \\
\text { diameter } \\
\mathrm{mm}\end{array}$ & $\begin{array}{c}\text { Lumen } \\
\text { volume } \\
\mathrm{mL}\end{array}$ & $\begin{array}{c}\text { Inflation } \\
\text { compliance } \\
\text { as strain }\end{array}$ & $\begin{array}{c}\text { Deflation } \\
\text { compliance } \\
\text { as strain }\end{array}$ \\
\hline 1 & 1.8 & 0.10 & 0.029 & 0.027 \\
2 & 2.2 & 0.05 & 0.192 & 0.214 \\
3 & 1.6 & 0.05 & 0.056 & 0.069 \\
4 & 1.6 & 0.01 & 0.090 & 0.086 \\
5 & & 0.03 & 0.066 & 0.076 \\
\hline Mean & & & 0.087 & 0.094 \\
SEM & & & 0.028 & 0.032
\end{tabular}

All human bronchi were from infants who died of sudden infant death syndrome (SIDS). Luminal volume $\left(V_{0}\right)$ is the volume of the bronchial segment lumen with no transmural pressure difference. Inflation and deflation compliances are specific compliances and refer to the slope of the normalized volume-pressure curves during inflation and deflation of the bronchi. Volume change $(\Delta V)$ was normalized as strain (i.e. $\Delta V / V 0)$. 
qualitative changes in struture were observed. Strainpressure curves for distal and proximal regions of stem bronchi were not significantly different, so regional differences in specific compliance cannot explain the increased specific compliance of the immature bronchi reported here.

Increased specific compliance of the immature bronchial wall would decrease both the preloads and afterloads on airway smooth muscle. A decrease in preload will alter the position of airway smooth muscle on its length-tension curve towards a shorter resting length, and higher transmural pressures may be required to stretch the muscle to optimum length [5]. In the adult (see [8]) airway smooth muscle is at optimum length at or near functional residual capacity (FRC); we hypothesize that in infants, smooth muscle will be shorter than optimum length at FRC. Human infants normally breathe at lung volumes greater than FRC [27]; as a consequence of this, bronchial smooth muscle may be stretching towards optimum length. In contrast, decreasing the afterload of the bronchial wall on airway smooth muscle will reduce the load against which the muscle contracts, increasing muscle shortening during bronchoconstriction $[6,10]$. Highly compliant immature bronchi may show increased airway narrowing compared to adults, due to reduced afterloads. This elevated airway narrowing could contribute to childhood asthma. Resting resistance of immature airways is also likely to increase as a consequence of increased specific compliance $[3,12]$. Infants may also experience significant maximum expiratory flow limitation because of their higher bronchial specific compliance.

The mechanism for age-related changes in compliance could involve either a change in composition of the wall (e.g. more cartilage) or a change in the mechanical properties of some wall component (e.g. stiffer cartilage). Changes in airway smooth muscle tone are unlikely to have influenced the compliance of bronchi measured in this paper. We investigated whether the increased stiffness of bronchi from 4 week old pigs was related to an increased amount of bronchial cartilage. Foetal pig bronchi had the same proportion of cartilage as 4 week olds but were significantly less compliant, implying that the maturational change in compliance was not explained by changes in the amount of bronchial cartilage. Similarly, the observed changes in total wall area do not explain the changes in compliance, as both foetal and 4 week olds have the same total wall area but different specific compliance.

Cartilage and smooth muscle stiffness are important determinants of the maturational change in tracheal compliance $[28,29]$, but in bronchi the structures determining specific compliance are unknown. Inflated and uninflated porcine bronchi were examined histologically to determine the region of the airway wall that deforms to accommodate the change in lumen volume. Both immature and adult bronchi showed a change in internal area $(\mathrm{Ai})$ with inflation. This change in $\mathrm{Ai}$ was less than expected from the pressure-volume curves, but within the $95 \%$ confidence intervals. No statistically significant change in the outer area (Ao) or total wall area (WAtot) of adult bronchi was detected with inflation. The small expected change in Ao and WAtot for adult bronchi coupled with the high variance of the mor- phometric data probably prevented any change being detected. Much of the variance in the morphological data is due to variability in the airway diameter and the inability to observe the same bronchus at the same place before and after inflation.

As with the adult, no difference in Ao was detected between inflated and uninflated bronchi from 1 week old pigs. If all of the change in $\mathrm{Ai}$ expected from the pressure-strain curves occurred by expansion of Ao, then a significant change $(\alpha<0.05)$ should have been detected more than $90 \%$ of the time $(\beta<0.1)$ This suggests that the cartilage layer may not be the only histological region controlling specific compliance for luminal changes in pressure. Inflation to $20 \mathrm{cmH}_{2} \mathrm{O}$ did produce significant reductions in the wall thickness and total wall area (WAtot) of 1 week old bronchi; therefore, at this pressure, part of the increase in lumen volume occurs by compression of the airway wall. Some expansion of Ao and movement of the cartilage plates may occur, but, at least in bronchi from immature pigs, airway wall compression is a factor in determining lumen volume and specific compliance.

Airway mucosa and smooth muscle are incompressible structures [30]; therefore, the region of wall compression may lie in the submucosa between the muscle and cartilage layers. This region can expand during muscle constriction in vitro, suggesting that it may also be compressible $[1,31]$. In vitro, fluid appears able to enter the wall of isolated bronchi across their adventitial surface (unpublished obervations) whether this can occur in the lung in vivo, is questionable. However, movement of interstitial fluid into or out of the airway wall could occur in vivo, via the vascular or lymphatic systems. So wall compression and interstitial fluid shifts could potentially influence airway distensibility in vivo. Whether any significant wall compression occurs in vivo, is unknown.

The present study demonstrated a significant maturation of bronchial specific compliance postnatally, but not during the last few weeks prenatally. The decrease in specific compliance with age was not caused by an increased amount of cartilage in the bronchial wall, and airway wall structures other than cartilage may be important in determining the compliance of immature bronchi.

Acknowledgements: The authors thank A. Light for technical help with histology and for developing the modified alcian blue and safranine stain used in this study. They also thank the Western Australian State Coroner and the staff of the forensic pathology service for help with obtaining human tissues.

\section{References}

1. Olsen CR, Stevens AE, Pride NB, Staub NC. Structural basis for decreased compressibility of constricted tracheae and bronchi. J Appl Physiol 1967; 23: 35-39.

2. Bhutani VK, Rubenstein D, Shaffer TH. Pressure-induced deformation in immature airways. Pediatr Res 1981; 15 : 829-832.

3. McCormack GS, Moreno RH, Hogg JC, Paré PD. Lung mechanics in papain-treated rabbits. J Appl Physiol 1986; 60: 242-246. 
4. Moreno RH, McCormack GS, Mullen JBM, Hogg JC, Bert J, Paré PD. Effect of intravenous papain on tracheal pressure-volume curves in rabbits. J Appl Physiol 1986; 60: 247-252.

5. Moreno RH, Paré PD. Intravenous papain-induced cartilage softening decreases preload of tracheal smooth muscle. J Appl Physiol 1989; 66: 1694-1698.

6. Jiang H, Stephens NL. Contractile properties of bronchial smooth muscle with and without cartilage. J Appl Physiol 1990; 69: 120-126.

7. Ishida K, Paré PD, Hards J, Schellenberg RR. Mechanical properties of human bronchial smooth muscle in vitro. J Appl Physiol 1992; 73: 1481-1485.

8. Moreno RH, Hogg JC, Paré PD. Mechanics of airway narrowing. Am Rev Respir Dis 1986; 133: 1171-1180.

9. Bramley AM, Thomson RJ, Robert CR, Schellenberg RR. Hypothesis: excessive bronchoconstriction in asthma is due to decreased airway elastance. Eur Respir J 1994; 7: 337-341.

10. Ishida K, Paré PD, Blogg T, Schellenberg RR. Effects of elastic loading on porcine trachealis muscle mechanics. J Appl Physiol 1990; 69: 1033-1039.

11. Dawson SV, Elliott EA. Wave speed limitation on expiratory flow: a unifying concept. J Appl Physiol: Respirat Environ Exercise Physiol 1977; 43: 498-515.

12. Jones JG, Fraser RB, Nadel JA. Effects of changing airway mechanics on maximum expiratory flow. $J$ Appl Physiol 1975; 38: 1012-1021.

13. Bhutani VK, Ritchie WG, Shaffer TH. Acquired tracheomegaly in very preterm neonates. Am J Dis Child 1986; 140: 449-452.

14. Martinez FD. Sudden infant death syndrome and small airway occlusion: facts and a hypothesis. Pediatrics 1991; 87: 190-198.

15. Southall DP. Role of apnea in the sudden infant death syndrome: a personal view. Pediatrics 1988; 80: 73-84.

16. Bhutani VK, Rubenstein SD, Shaffer TH. Pressure-volume relationships of tracheae in foetal newborn and adult rabbits. Respir Physiol 1981; 43: 221-231.

17. Bhutani VK, Koslo RJ, Shaffer TH. The effect of tracheal smooth muscle tone on neonatal airway collapsibility. Pediatr Res 1986; 20: 492-495.

18. Croteau JR, Cook CD. Volume-pressure and length-ten- sion measurements in human tracheal and bronchial segments. J Appl Physiol 1961; 16: 170-172.

19. Mitchell HW, McFawn PK, Sparrow MP. Increased narrowing of bronchial segments from immature pigs. Eur Respir J 1992; 5: 207-212.

20. Mitchell HW, Willet KE, Sparrow MP. Perfused bronchial segment and bronchial strip: narrowing $v s$ isometric force by mediators. J Appl Physiol 1989; 66: 2704-2709.

21. Omari TI, Sparrow MP. Epithelial disruption by proteases augments the responsiveness of porcine bronchial segments. Clin Exp Pharmacol Physiol 1992; 19: 785-794.

22. Mitchell HW, Sparrow MP, Tagliaferri RP. Inhibitory and excitatory responses to field stimulation in foetal and adult pig airway. Pediatr Res 1990; 28: 69-74.

23. Gunst SJ, Stropp JQ. Pressure-volume and length-stress relationships in canine bronchi in vitro. J Appl Physiol 1988; 64: 2522-2531.

24. Bai A, Eidelman DH, Hogg JC, et al. Proposed nomenclature for quantifying subdivisions of the bronchial wall. J Appl Physiol 1994; 77: 1011-1014.

25. Feldman HA. Families of lines: random effects in linear regression analysis. J Appl Physiol 1988; 64: 1721-1732.

26. Karlinsky JB, Snider GL, Franzblau C, Stone PJ, Hoppin FG. In vitro effects of elastase and collagenase on mechanical properties of hamster lungs. Am Rev Respir Dis 1976; 113: 769-776.

27. Mortola JP. Physiology of postnatal growth. In: Crystal RG, West JB, eds. The Lung: Scientific Foundations. New York, Raven Press Ltd, 1991; pp. 1735-1748.

28. Penn RB, Wolfson MR, Shaffer TH. Developmental differences in tracheal cartilage mechanics. Pediatr Res 1989; 26: 429-433.

29. Panitch HB, Deoras KS, Wolfson MR, Shaffer TH. Maturational changes in airway smooth muscle structure-function relationships. Pediatr Res 1992; 31: 151-156.

30. James AL, Hogg JC, Dunn LA, Paré PD. The use of internal perimeter to compare airway size and to calculate smooth muscle shortening. Am Rev Respir Dis 1988; 138: 135-139.

31. Mitchell HW, Sparrow MP. Videoimaging of lumen narrowing: muscle shortening and flow responsiveness in isolated bronchial segments of the pig. Eur Respir $J$ 1994; 7: 1317-1325. 